On the arithmetical rank of an intersection of ideals

\title{
On the arithmetical rank of an intersection of ideals $^{1}$
}

\author{
Margherita Barile ${ }^{2}$ \\ Dipartimento di Matematica, Università di Bari, Via E. Orabona 4, \\ 70125 Bari, Italy ${ }^{3}$
}

\begin{abstract}
We determine sets of elements which, under certain conditions, generate an intersection of ideals up to radical.

Keywords: Arithmetical rank, monomial ideals, ideal intersection.

\section{Introduction}

Let $R$ be Noetherian commutative ring with identity. We say that some elements $\gamma_{1}, \ldots, \gamma_{r} \in R$ generate an ideal $I$ of $R$ up to radical if $\sqrt{I}=\sqrt{\left(\gamma_{1}, \ldots, \gamma_{r}\right)}$. The smallest $r$ with this property is called the arithmetical rank of $I$, denoted ara $I$. It is well known that height $I \leq \operatorname{ara} I$. If equality holds, $I$ is called a set-theoretic complete intersection (s.t.c.i.). Determining the arithmetical rank of an ideal $I$, or at least a satisfactory upper bound for it, is, in general, a hard task. In this paper we give some criteria for the case where $I$ is the intersection of two ideals, whose generators are linked by special divisibility conditions. Our results yield constructive methods which can be applied to certain polynomial ideals generated by squarefree monomials. In particular, we characterize a class of monomial ideals of minimal multiplicity which are s.t.c.i..

Other results on the arithmetical rank of intersections of ideals can be found in [1.
\end{abstract}

\section{The Main Theorem}

For the proof of our main theorem we will need the following preliminary result, which was presented in [3, and is a generalization of the lemma in [11, p. 249.

Lemma 1 Let $P_{1}, \ldots, P_{r}$ be finite subsets of $R$, and set $P=\bigcup_{i=1}^{r} P_{i}$. Suppose that

(i) $P_{1}$ has exactly one element;

(ii) if $p$ and $p^{\prime}$ are different elements of $P_{i}(1<i \leq r)$ then $\left(p p^{\prime}\right)^{m} \in$ $\left(\bigcup_{j=1}^{i-1} P_{j}\right)$ for some positive integer $m$.

\footnotetext{
${ }^{1} \mathrm{MSC}$ 2000: 13A15; 13F55, 14M10.

${ }^{2}$ Partially supported by the Italian Ministry of Education, University and Research.

3e-mail: barile@dm.uniba.it, Fax:0039080 5963612
} 
We set $q_{i}=\sum_{p \in P_{i}} p^{e(p)}$, where $e(p) \geq 1$ are arbitrary integers. Then we get

$$
\sqrt{(P)}=\sqrt{\left(q_{1}, \ldots, q_{r}\right)}
$$

Before stating our main result, we introduce the following notation: given $\alpha_{1}, \ldots, \alpha_{t}, \beta_{1}, \ldots, \beta_{t} \in R$, we write $\left[\alpha_{1}, \ldots, \alpha_{t}\right] \subset\left[\beta_{1}, \ldots, \beta_{t}\right]$ if, for all indices $i$ such that $1 \leq i \leq t,\left(\alpha_{1}, \ldots, \alpha_{i}\right) \subset\left(\beta_{1}, \ldots, \beta_{i}\right)$.

Theorem 1 Let $\mu_{1}, \ldots, \mu_{u}, \nu_{1}, \ldots, \nu_{v}$ be elements of $R$ and consider the ideals $I=\left(\mu_{1}, \ldots, \mu_{u}\right)$, and $J=\left(\nu_{1}, \ldots, \nu_{v}\right)$ of $R$. Suppose that there is a positive integer $s$ with $s<u$ and $s \leq v$ such that

$$
\left[\mu_{1}, \ldots, \mu_{s}\right] \subset\left[\nu_{1}, \ldots, \nu_{s}\right]
$$

For $1 \leq i \leq u-s$ set

$$
\gamma_{i}=\sum_{j=s+1}^{s+i} \mu_{j} \nu_{i-j+s+1}
$$

and, for $u-s+1 \leq i \leq u$, set

$$
\gamma_{i}=\mu_{i-u+s}+\sum_{j=s+1}^{s+i} \mu_{j} \nu_{i-j+s+1}
$$

If $s \leq v-2$, for $u+1 \leq i \leq u+v-s-1$, set further

$$
\gamma_{i}=\sum_{j=s+1}^{s+i} \mu_{j} \nu_{i-j+s+1}
$$

In equations (2), (3) and (4) we have adopted the following convention: we have set $\mu_{h}=0$ whenever $h>u$ and $\nu_{k}=0$ whenever $k>v$.

Then, if $s \leq v-2$,

$$
\sqrt{I \cap J}=\sqrt{\left(\gamma_{1}, \ldots, \gamma_{u+v-s-1}\right)}
$$

and, if $v-1 \leq s \leq v$,

$$
\sqrt{I \cap J}=\sqrt{\left(\gamma_{1}, \ldots, \gamma_{u}\right)}
$$

Note 1 We can rewrite (2) and (4) in the form:

$$
\gamma_{i}=\sum_{\substack{h+k=i+s+1 \\ h \geq s+1}} \mu_{h} \nu_{k}, \quad(i \leq u-s \text { or } i \geq u+1)
$$

and (3) in the form

$$
\gamma_{i}=\mu_{i-u+s}+\sum_{\substack{h+k=i+s+1 \\ h \geq s+1}} \mu_{h} \nu_{k} . \quad(u-s+1 \leq i \leq u)
$$

We are going to use this simplified notation in the proof of Theorem 1 
Proof .-We prove the equalities (5) and (6) by showing the two inclusions. For all indices $i$, all summands of $\gamma_{i}$ belong to $\sqrt{I \cap J}$ : this is certainly true for the terms of the form $\mu_{h} \nu_{k}$; moreover, for $u-s+1 \leq i \leq u$, we have that $1 \leq i-u+s \leq s$, so that, by (11), $\mu_{i-u+s} \in I \cap J$. Thus inclusion $\supset$ is true. We now prove $\subset$. For all indices $i$, let $P_{i}$ be the set of summands of $\gamma_{i}$. Here we are excluding the terms containing $\mu_{h}$ with $h>u$ or $\nu_{k}$ with $k>v$. We show that the sets $P_{i}$ fulfill the assumption of Lemma 11 Since $P_{1}=\left\{\mu_{s+1} \nu_{1}\right\}$, (i) is satisfied. Now let $i \geq 2$ and let $p$ and $p^{\prime}$ be two distinct elements of $P_{i}$. Then one of the following two cases occurs:

(a) $p=\mu_{h} \nu_{k}$ and $p^{\prime}=\mu_{h^{\prime}} \nu_{k^{\prime}}$, where, without loss of generality, we may assume that $1 \leq k^{\prime}<k$. Let $l=k-k^{\prime}$. Note that $p p^{\prime}$ is divisible by $p^{\prime \prime}=\mu_{h} \nu_{k^{\prime}}$. Since $h+k=i+s+1$, and $h \geq s+1$, we have $k \leq i$, so that $l<i$. Moreover, $h+k^{\prime}=h+k-l=i-l+s+1$, so that $p^{\prime \prime} \in P_{i-l}$, and, consequently, $p p^{\prime} \in P_{i-l}$.

(b) $u-s+1 \leq i \leq u$ and $p=\mu_{i-u+s}, p^{\prime}=\mu_{h^{\prime}} \nu_{k^{\prime}}$. Then $1 \leq i-u+s \leq s$, so that, by (1), $p p^{\prime} \in\left(\nu_{1}, \ldots, \nu_{i-u+s}\right)\left(\mu_{h^{\prime}} \nu_{k^{\prime}}\right)$. Moreover, for $1 \leq k \leq i-u+s$, $\mu_{h^{\prime}} \nu_{k} \in P_{h^{\prime}+k-s-1}$, where $h^{\prime}+k-s-1 \leq h^{\prime}+i-u-1 \leq u+i-u-1=i-1$. Hence $p p^{\prime} \in\left(\cup_{j=1}^{i-1} P_{j}\right)$.

This shows that (ii) is fulfilled, too. It follows that

$$
\sqrt{(P)}= \begin{cases}\sqrt{\left(\gamma_{1}, \ldots, \gamma_{u+v-s-1}\right)} & \text { if } s \leq v-2 \\ \sqrt{\left(\gamma_{1}, \ldots, \gamma_{u}\right)} & \text { if } v-1 \leq s \leq v .\end{cases}
$$

Now it remains to show that

$$
\sqrt{I \cap J} \subset \sqrt{(P)}
$$

Recall that $\sqrt{I \cap J}=\sqrt{I J}$, and $I J$ is generated by all products $\mu_{h} \nu_{k}$, with $1 \leq h \leq u, 1 \leq k \leq v$. If $h \geq s+1$, then $\mu_{h} \nu_{k} \in P_{h+k-s-1}$ : note that $h+k-s-1 \leq u+v-s-1$. If $1 \leq h \leq s$, then $\mu_{h} \in P_{h+u-s}$. In either case, $\mu_{h} \nu_{k} \in(P)$. This shows (7) and completes the proof.

As an immediate consequence of Theorem 1 we have:

Corollary 1 Let $\mu_{1}, \ldots, \mu_{u}, \nu_{1}, \ldots, \nu_{v}$ be elements of $R$ and consider the ideals $I=\left(\mu_{1}, \ldots, \mu_{u}\right)$, and $J=\left(\nu_{1}, \ldots, \nu_{v}\right)$ of $R$. Suppose that there is a positive integer $s$ with $s<u$ and $s \leq v$ such that $\left[\mu_{1}, \ldots, \mu_{s}\right] \subset\left[\nu_{1}, \ldots, \nu_{s}\right]$. Then

$$
r=\operatorname{ara}(I \cap J) \leq \begin{cases}u+v-s-1 & \text { if } s \leq v-2, \\ u & \text { if } v-1 \leq s \leq v .\end{cases}
$$

Moreover, the elements $\gamma_{1}, \ldots, \gamma_{r} \in R$ generating $I \cap J$ up to radical can be chosen in such a way that

$$
\left[\gamma_{1}, \ldots, \gamma_{u}\right] \subset\left[\mu_{s+1}, \ldots, \mu_{u}, \mu_{1}, \ldots, \mu_{s}\right]
$$

and

$$
\left[\gamma_{1}, \ldots, \gamma_{v}\right] \subset\left[\nu_{1}, \ldots, \nu_{v}\right]
$$


Proof .-We only prove statement (9). For all indices $i$ such that $1 \leq i \leq u-s$ from (2) we deduce that $\gamma_{i} \in\left(\nu_{1} \ldots, \nu_{i}\right)$. Now let $u-s+1 \leq i \leq u$. Then, by (3),

$$
\gamma_{i} \in\left(\mu_{i-u+s}, \nu_{1}, \ldots, \nu_{i}\right)
$$

where $1 \leq i-u+s \leq s$, and, moreover, $i-u+s<i$. Hence, in view of (11), $\mu_{i-u+s} \in\left(\nu_{1}, \ldots, \nu_{i}\right)$, so that $\gamma_{i} \in\left(\nu_{1}, \ldots, \nu_{i}\right)$. If $v \leq u$, this yields the claim. Otherwise, since $v \leq u+v-s-1$, for all indices $i$ with $u+1 \leq i \leq v$, by (4) we have that $\gamma_{i} \in\left(\nu_{1}, \ldots, \nu_{i}\right)$. This completes the proof of (9). Statement (8) can be shown in a similar way.

Remark 1 The claim of Corollary 1 also holds for $s=0$ : it is well known that, whenever $I$ and $J$ are ideals of $R$ generated by $u$ and $v$ elements respectively, then

$$
r=\operatorname{ara}(I \cap J) \leq u+v-1 .
$$

A proof of this result and its generalization to the intersection of any finite number of ideals is given in [11, Theorem 1.

Example 1 Let $K$ be a field. Consider the following ideal of $R=K\left[x_{1}, \ldots, x_{5}\right]$ :

$I=\left(x_{1}, x_{3}, x_{5}\right) \cap\left(x_{2}, x_{4}, x_{5}\right) \cap\left(x_{1}, x_{4}, x_{5}\right) \cap\left(x_{2}, x_{3}, x_{5}\right) \cap\left(x_{1}, x_{3}, x_{6}\right) \cap\left(x_{2}, x_{5}, x_{7}\right)$,

which is of pure height 3 . We have $I=\left(x_{1} x_{2}, x_{1} x_{5}, x_{3} x_{5}, x_{5} x_{6}, x_{3} x_{4} x_{7}, x_{2} x_{3} x_{4}\right)$. We show that ara $I=3$, i.e., $I$ is a s.t.c.i.. Note that $I=I_{1} \cap I_{2} \cap I_{3}$, where

$$
I_{1}=\left(x_{1} x_{2}, x_{3} x_{4}, x_{5}\right), I_{2}=\left(x_{1}, x_{3}, x_{6}\right), I_{3}=\left(x_{2}, x_{5}, x_{7}\right) .
$$

First set

$$
\begin{array}{ll}
\mu_{1}=x_{1} x_{2} & \nu_{1}=x_{1} \\
\mu_{2}=x_{3} x_{4} & \nu_{2}=x_{3} \\
\mu_{3}=x_{5} & \nu_{3}=x_{6} .
\end{array}
$$

Then Corollary 1 applies to $I_{1} \cap I_{2}$ with $u=v=3, s=2$ and $r=3$. Hence $I_{1} \cap I_{2}$ is generated up to radical by the following 3 elements:

$$
\gamma_{1}=x_{1} x_{5}, \gamma_{2}=x_{1} x_{2}+x_{3} x_{5}, \gamma_{3}=x_{3} x_{4}+x_{5} x_{6} .
$$

Now set

$$
\begin{array}{ll}
\mu_{1}^{\prime}=\gamma_{1} & \nu_{1}^{\prime}=x_{5} \\
\mu_{2}^{\prime}=\gamma_{2} & \nu_{2}^{\prime}=x_{2} \\
\mu_{3}^{\prime}=\gamma_{3} & \nu_{3}^{\prime}=x_{7} .
\end{array}
$$

Then Corollary 1 applies to the intersection of the ideals $\left(\gamma_{1}, \gamma_{2}, \gamma_{3}\right)$ and $I_{3}$ with $u=v=3$ and $s=2$. Thus this intersection is generated up to radical by

$$
\begin{aligned}
\gamma_{1}^{\prime} & =\left(x_{3} x_{4}+x_{5} x_{6}\right) x_{5} \\
\gamma_{2}^{\prime} & =x_{1} x_{5}+\left(x_{3} x_{4}+x_{5} x_{6}\right) x_{2} \\
\gamma_{3}^{\prime} & =x_{1} x_{2}+x_{3} x_{5}+\left(x_{3} x_{4}+x_{5} x_{6}\right) x_{7}
\end{aligned}
$$

These elements generate $I$ up to radical. 
Example 2 In the polynomial ring $R=K\left[x_{1}, \ldots, x_{6}\right]$ consider the ideals $I_{1}=$ $\left(x_{1}, x_{5}, x_{6}\right) \cap\left(x_{4}, x_{5}, x_{6}\right)=\left(x_{1} x_{4}, x_{5}, x_{6}\right)$ and $I_{2}=\left(x_{1}, x_{2}, x_{3}\right)$ and set $I=$ $I_{1} \cap I_{2}=\left(x_{1} x_{4}, x_{1} x_{5}, x_{1} x_{6}, x_{2} x_{5}, x_{2} x_{6}, x_{3} x_{5}, x_{3} x_{6}\right)$. Then Corollary 1 applies to $I_{1} \cap I_{2}$ with $u=v=3$ and $s=1$, so that $I$ is generated, up to radical, by

$$
\begin{aligned}
\gamma_{1} & =x_{1} x_{5} \\
\gamma_{2} & =x_{2} x_{5}+x_{1} x_{6}, \\
\gamma_{3} & =x_{1} x_{4}+x_{3} x_{5}+x_{2} x_{6}, \\
\gamma_{4} & =x_{3} x_{6} .
\end{aligned}
$$

Thus ara $I \leq 4$. We show that equality holds: to this end we exploit the inequality

$$
\operatorname{cd} I \leq \operatorname{ara} I
$$

(see 9], Theorem 3.4, or [8], Example 2, pp. 414-415), where cd denotes the so-called local cohomological dimension of $I$, which is defined as follows:

$$
\operatorname{cd} I=\max \left\{i \mid H_{I}^{i}(R) \neq 0\right\} .
$$

We prove that $H_{I}^{4}(R) \neq 0$. This will imply that $4 \leq \operatorname{cd} I \leq$ ara $I \leq 4$, so that equality holds everywhere. In particular, $I$ is not a s.t.c.i.. We have the following Mayer-Vietoris sequence (see [9], p. 15):

$$
\cdots \rightarrow H_{I_{1}}^{4}(R) \oplus H_{I_{2}}^{4}(R) \rightarrow H_{I_{1} \cap I_{2}}^{4}(R) \rightarrow H_{I_{1}+I_{2}}^{5}(R) \rightarrow H_{I_{1}}^{5}(R) \oplus H_{I_{2}}^{5}(R) \rightarrow \cdots
$$

In view of (10), since $I_{1}$ and $I_{2}$ are generated by three elements, we have that $H_{I_{1}}^{i}(R)=H_{I_{2}}^{i}(R)=0$ for all $i>3$. Moreover, $I_{1}+I_{2}=\left(x_{1}, x_{2}, x_{3}, x_{5}, x_{6}\right)$ is generated by a regular sequence of length 5 , so that by [8], Example 2, pp. 414415, cd $I_{1}+I_{2}=5$ and, on the other hand, by [9], Proposition 2.8, $H_{I_{1}+I_{2}}^{i}(R)=0$ for all $i<5$. Thus $H_{I_{1}+I_{2}}^{i}(R) \neq 0$ if and only if $i=5$. From (11) it thus follows that $H_{I_{1} \cap I_{2}}^{4}(R) \neq 0$, as required.

Example 3 In any commutative ring $R$ with identity consider the ideal

$$
I=\left(\mu_{1}, \mu_{2}, \mu_{3}\right) \cap\left(\nu_{1}, \nu_{2}, \nu_{3}\right) \cap\left(\xi_{1}, \xi_{2}, \xi_{3}\right),
$$

where we assume that $\mu_{1} \in\left(\nu_{1}\right)$ and $\mu_{2} \in\left(\xi_{1}\right)$. Corollary 1 applies to the ideal $I^{\prime}=\left(\mu_{1}, \mu_{2}, \mu_{3}\right) \cap\left(\nu_{1}, \nu_{2}, \nu_{3}\right)$ with $u=v=3$ and $s=1$. Hence, according to (8), it is generated, up to radical, by 4 elements $\gamma_{1}, \gamma_{2}, \gamma_{3}, \gamma_{4} \in R$ such that $\gamma_{1} \in\left(\mu_{2}\right)$; consequently, $\gamma_{1} \in\left(\xi_{1}\right)$. Thus $I^{\prime \prime}=\left(\gamma_{1}, \gamma_{2}, \gamma_{3}, \gamma_{4}\right) \cap\left(\xi_{1}, \xi_{2}, \xi_{3}\right)$ fulfills the assumption of Corollary 1 for $u=4, v=3$ and $s=1$. Note that $\sqrt{I^{\prime \prime}}=\sqrt{I}$. Therefore ara $I \leq 5$. For instance, in the polynomial ring $K\left[x_{1}, \ldots, x_{10}\right]$, where $K$ is a field, consider the ideal

$$
I=\left(x_{1} x_{4}, x_{2} x_{5}, x_{3} x_{6}\right) \cap\left(x_{1}, x_{7}, x_{8}\right) \cap\left(x_{2}, x_{9}, x_{10}\right) .
$$


It is generated, up to radical, by the following 5 polynomials:

$$
\begin{aligned}
& \left(x_{2} x_{5} x_{7}+x_{1} x_{3} x_{6}\right) x_{2}, \\
& \left(x_{2} x_{5} x_{7}+x_{1} x_{3} x_{6}\right) x_{9}+\left(x_{1} x_{4}+x_{2} x_{5} x_{8}+x_{3} x_{6} x_{7}\right) x_{2}, \\
& \left(x_{2} x_{5} x_{7}+x_{1} x_{3} x_{6}\right) x_{10}+\left(x_{1} x_{4}+x_{2} x_{5} x_{8}+x_{3} x_{6} x_{7}\right) x_{9}+x_{2} x_{3} x_{6} x_{8}, \\
& x_{1} x_{2} x_{5}+\left(x_{1} x_{4}+x_{2} x_{5} x_{8}+x_{3} x_{6} x_{7}\right) x_{10}+x_{3} x_{6} x_{8} x_{9}, \\
& x_{3} x_{6} x_{8} x_{10} .
\end{aligned}
$$

As was proven in [10, the cohomological dimension of a monomial ideal is equal to its projective dimension (pd). In our case, a computation with $\mathrm{CoCoA}$ [ yields that $\operatorname{pd} I=5$ if $\operatorname{char} K=0$. Hence, in view of (10), we have that ara $I=5$ in characteristic zero. Another computation with CoCoA yields that the minimum number of generators of $I$ is 15 .

\section{An application}

In this section we apply the results of the previous section to the computation of the arithmetical ranks of a certain class of ideals generated by monomials in a polynomial ring over a field $K$. Since the arithmetical rank is the same up to radical, without loss of generality, we can restrict our attention to ideals generated by squarefree monomials. We first prove one general result.

Proposition 1 Let $h, t$ be integers, $h \leq t$, and let $a_{0}, a_{1}, \ldots, a_{h}$ be integers with $0=a_{0}<a_{1}<\cdots<a_{h} \leq t$. Let $x_{1}, \ldots, x_{t}, y_{1}, \ldots, y_{a_{h}} \in R$ and consider the following ideals of $R$ :

$$
I_{0}=\left(x_{1}, \ldots, x_{t}\right),
$$

and, for all indices $i$ with $1 \leq i \leq h$,

$$
I_{i}=\left(y_{1}, \ldots, y_{a_{i}}, x_{a_{i}+1}, \ldots, x_{t}\right) .
$$

Then there are $\gamma_{1}, \ldots, \gamma_{t+a_{h}-h} \in R$ generating $I_{0} \cap I_{1} \cap \cdots \cap I_{h}$ up to radical such that

$$
\left[\gamma_{1}, \ldots, \gamma_{a_{h}}\right] \subset\left[y_{1}, \ldots, y_{a_{h}}\right],
$$

and

$$
\gamma_{i}=x_{i-a_{h}+h}
$$

for all indices $i$ with $2 a_{h}-h+1 \leq i \leq t+a_{h}-h$.

Proof .-We proceed by induction on $h$. The claim is trivially true for $h=0$. Suppose that $h \geq 1$ and that the claim is true for all smaller indices. There are $\bar{\gamma}_{1}, \ldots, \bar{\gamma}_{t+a_{h-1}-h+1} \in R$ generating $I_{0} \cap I_{1} \cap \cdots \cap I_{h-1}$ up to radical such that

$$
\left[\gamma_{1}, \ldots, \gamma_{a_{h-1}}\right] \subset\left[y_{1}, \ldots, y_{a_{h-1}}\right],
$$

and

$$
\gamma_{i}=x_{i-a_{h-1}+h-1}
$$


for all indices $i$ with $2 a_{h-1}-h+2 \leq i \leq t+a_{h-1}-h+1$. Note that

$$
\begin{aligned}
& \sqrt{I_{0} \cap \cdots \cap I_{h-1} \cap I_{h}} \\
= & \sqrt{\left(\bar{\gamma}_{1}, \cdots, \bar{\gamma}_{t+a_{h-1}-h+1}\right) \cap\left(y_{1}, \ldots, y_{a_{h}}, x_{a_{h}+1}, \ldots, x_{t}\right)} \\
= & \sqrt{\left(\bar{\gamma}_{1}, \ldots, \bar{\gamma}_{a_{h}+a_{h-1}-h+1}, x_{a_{h}+1}, \ldots, x_{t}\right) \cap\left(y_{1}, \ldots, y_{a_{h}}, x_{a_{h}+1}, \ldots, x_{t}\right)} \\
& \left(\text { note that } a_{h}+a_{h-1}-h+1 \geq 2 a_{h-1}-h+2\right) \\
= & \sqrt{\left(\bar{\gamma}_{1}, \cdots, \bar{\gamma}_{a_{h}+a_{h-1}-h+1}\right) \cap\left(y_{1}, \ldots, y_{a_{h}}\right)+\left(x_{a_{h}+1}, \ldots, x_{t}\right) .}
\end{aligned}
$$

In view of (13), Corollary 1 applies to $\left(\bar{\gamma}_{1}, \cdots, \bar{\gamma}_{a_{h}+a_{h-1}-h+1}\right) \cap\left(y_{1}, \ldots, y_{a_{h}}\right)$ for $u=a_{h}+a_{h-1}-h+1, v=a_{h}$ and $s=a_{h-1}$. Hence, by (9), this ideal is generated up to radical by $\gamma_{1}, \ldots, \gamma_{2 a_{h}-h} \in R$ such that

$$
\left[\gamma_{1}, \ldots, \gamma_{a_{h}}\right] \subset\left[y_{1}, \ldots, y_{a_{h}}\right]
$$

Set $\gamma_{i}=x_{i-a_{h}+h}$ for all indices $i$ such that $2 a_{h}-h+1 \leq i \leq t+a_{h}-h$. Then, by (14), $\gamma_{1}, \ldots, \gamma_{t+a_{h}-h}$ generate $I_{0} \cap \cdots \cap I_{h-1} \cap I_{h}$ up to radical. This completes the proof.

Corollary 2 Let $K$ be a field and let $x_{1}, \ldots, x_{t}, y_{1}, \ldots, y_{t}$ be pairwise distinct indeterminates over $K$. In the polynomial ring $R=K\left[x_{1}, \ldots, x_{t}, y_{1}, \ldots, y_{t}\right]$ consider the ideal

$$
I=\left(x_{1}, \ldots, x_{t}\right) \cap\left(y_{1}, x_{2}, \ldots, x_{t}\right) \cap \cdots \cap\left(y_{1}, \ldots, y_{t-1}, x_{t}\right) \cap\left(y_{1}, \ldots, y_{t}\right) .
$$

Then $I$ is a s.t.c.i..

Proof .-It suffices to apply Proposition 1 for $h=t$ (which implies that $a_{i}=i$ for all $i=1, \ldots, t)$. This yields ara $I \leq t$. Since height $I=t$, equality holds. This completes the proof.

Remark 2 Recall that the ideals generated by squarefree monomials in a polynomial ring over a field are the face ideals of simplicial complexes: we refer to Bruns and Herzog [5], Section 5, for the basic notions on this topic. The ideal $I$ in the claim of Corollary 2 is the face ideal of the simplicial complex $\Delta$ on the vertices $x_{1}, \ldots, x_{t}, y_{1}, \ldots, y_{t}$ whose facets are

$$
\begin{aligned}
F_{0} & =\left\{y_{1}, \ldots, y_{t}\right\}, \\
F_{1} & =\left\{x_{1}, y_{2}, \ldots, y_{t}\right\}, \\
\vdots & \\
F_{i-1} & =\left\{x_{1}, \ldots, x_{i-1}, y_{i}, \ldots, y_{t}\right\} \\
F_{i} & =\left\{x_{1}, \ldots, x_{i}, y_{i+1}, \ldots, y_{t}\right\}, \\
\vdots & \\
F_{t} & =\left\{x_{1}, \ldots, x_{t}\right\} .
\end{aligned}
$$


For all indices $i>0$,

$$
F_{i} \cap\left(\cup_{i=0}^{i-1} F_{i}\right)=\left\{x_{1}, \ldots, x_{i-1}, y_{i+1}, \ldots, y_{t}\right\},
$$

which is a maximal proper subset of $F_{i-1}$. According to [5], Definition 5.1.11., $\Delta$ is a so-called shellable complex. By virtue of [5], Theorem 5.1.13., as a consequence, we have that $R / I$ is a Cohen-Macaulay ring. By the results in [4, $I$ also defines a reducible variety of minimal degree according the classification given in [7, Section 4. Another class of varieties of minimal degree which are s.t.c.i. (and whose defining ideal is generated by a set formed of both monomials and binomials of degree two) has been recently characterized in [2.

\section{References}

[1] Barile, M., On the computation of arithmetical ranks, Int. J. Pure Appl. Math., 17, (2004), 143-161.

[2] Barile, M., Certain minimal varieties are set-theoretic complete intersections. Preprint (2005). math.AG/0509475 To appear in: Comm. Algebra.

[3] Barile, M., On ideals generated by monomials and one binomial. Preprint (2005). math.AC/0510030 To appear in: Algebra Colloq.

[4] Barile, M.; Morales, M., On the equations defining minimal varieties, Comm. Algebra, 28, (2000), 1223-1239.

[5] Bruns, W.; Herzog, J., Cohen-Macaulay Rings. Cambridge University Press. Cambridge, 1993.

[6] CoCoATeam, CoCoA, a system for doing Computations in Commutative Algebra. Available at http://cocoa.dima.unige.it.

[7] Eisenbud, D.; Goto, S., Linear free resolutions and minimal multiplicity. J. Algebra, 88, (1984), 89-133.

[8] Hartshorne, R., Cohomological dimension of algebraic varieties, Ann. of Math., 88, (1989), 403-450.

[9] Huneke, C. Lectures on local cohomology (with an appendix by Amelia Taylor). Available at http://www.math.ku.edu/ ${ }^{\sim}$ huneke/Vita/chicago-lc.pdf, 2004.

[10] Lyubeznik, G., On the local cohomology modules $H_{\mathcal{A}}^{i}(R)$ for ideals $\mathcal{A}$ generated by monomials in an $R$-sequence. In: Complete Intersections, Lectures given at the $1^{\text {st }} 1983$ Session of the Centro Internazionale Matematico Estivo (C.I.M.E.), Acireale, Italy, June 13-21, 1983; Greco, S., Strano, R., Eds.; Springer: Berlin Heidelberg, 1984.

[11] Schmitt, Th.; Vogel, W., Note on Set-Theoretic Intersections of Subvarieties of Projective Space, Math. Ann., 245, (1979), 247-253. 